\title{
13. PRELIMINARY RESULTS FROM SPECTROSCOPIC OBSERVATIONS OF FAINT B STARS
}

\author{
R. M. Petrie*
}

Dominion Astrophysical Observatory

Radial velocities of $570 \mathrm{~B}$ stars, mostly fainter than apparent magnitude $7 \cdot 5$ of spectral types between 08 and B5, have recently been determined at Victoria (Petrie and Pearce 1962). Spectroscopic absolute magnitudes based upon the measured strength of $\mathrm{H}_{\gamma}$ absorption have been determined for these stars. In addition to this totally new material, spectroscopic absolute magnitudes have been measured for an additional 180 stars. From the two lists 490 stars are available for

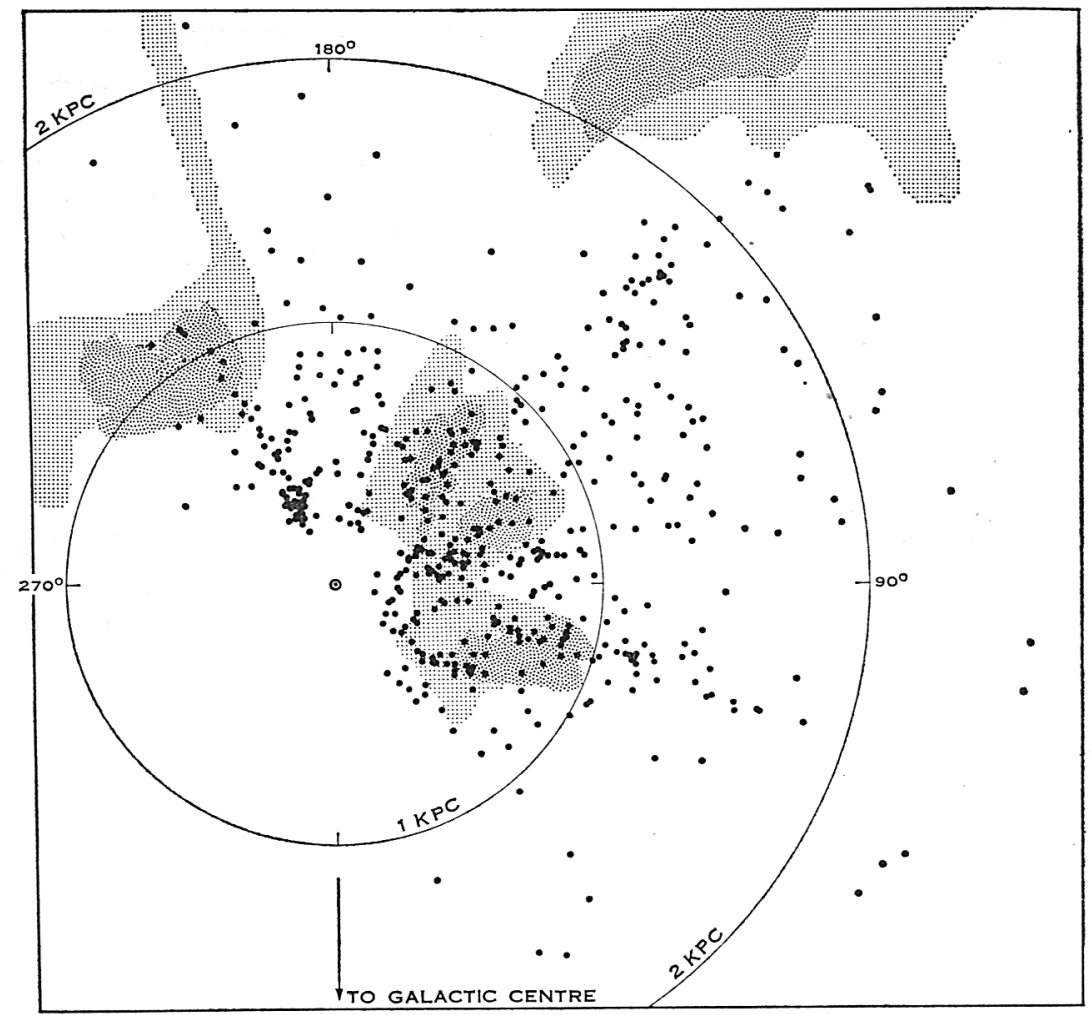

Fig. 1.-Distribution of O-B6 stars, projected on the galactic plane.

a study of their distributions and radial velocities, since for this number magnitudes and colours have been measured with sufficient precision to allow reliable estimates of the interstellar extinction and the true distance moduli. The results given here are preliminary in the sense that approximately 200 additional stars will be added to the study when the program to determine photoelectric magnitudes and colours

\footnotetext{
* Presented by J. F. Heard.
} 
is completed. The radial velocities and absolute magnitudes of all the stars have already been measured.

The distribution of the stars, projected on the galactic plane, is shown in Figure 1. A satisfactory distribution in distance exists to about $1.5 \mathrm{kpc}$ from the Sun; beyond this relatively few stars are found on our program except at certain longitudes. The distribution in longitude is confined largely to the hemisphere between galactic longitudes (new system) 35 and $215^{\circ}$. The positions, according to Kerr (1962), of the most dense clouds of neutral hydrogen are shown in Figure 1. There does not appear to be a close connection between the neutral hydrogen and the B stars of our program. Nor do these stars appear to delineate spiral structure although this may be a result of their relatively small distances from the Sun.

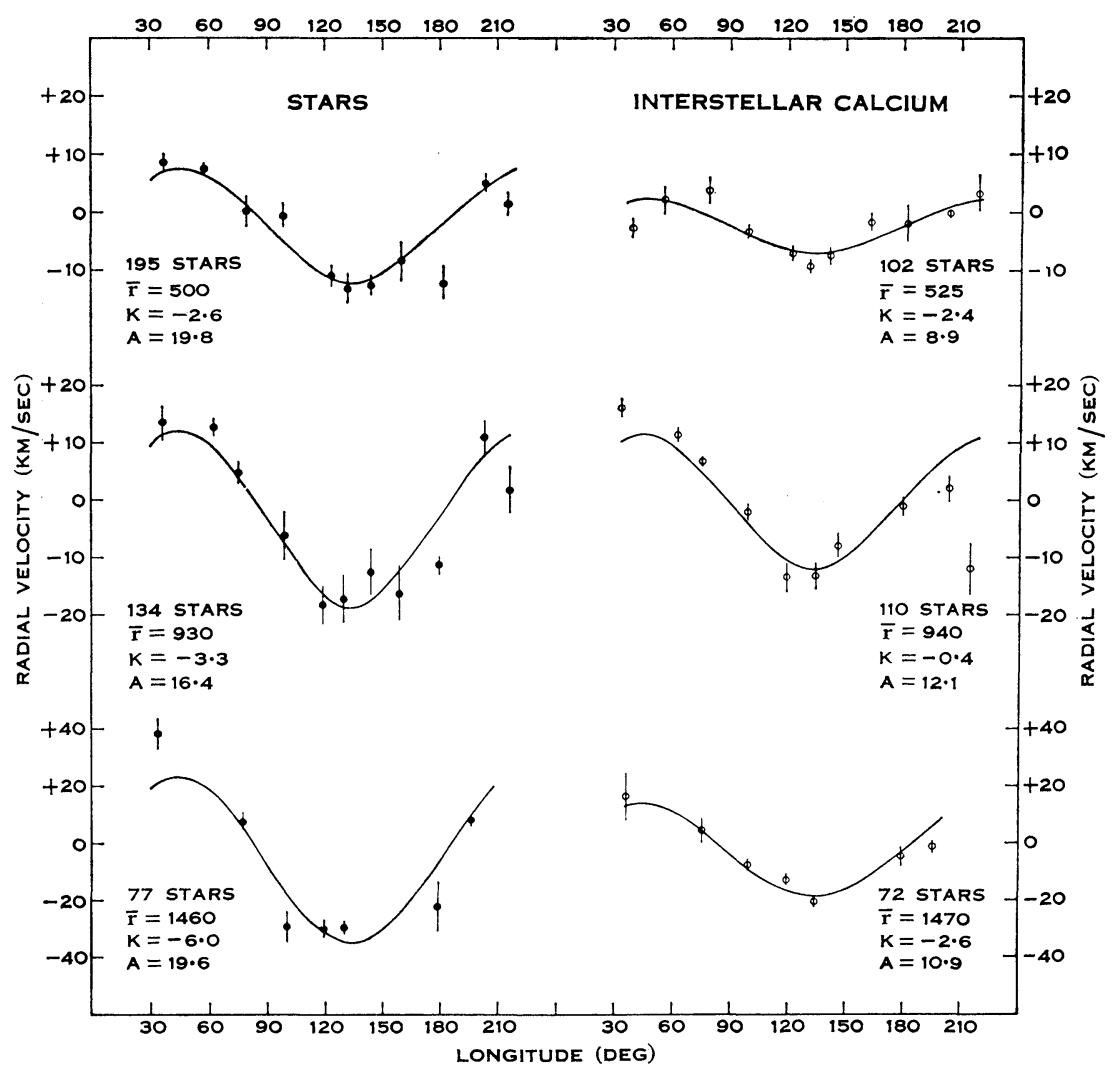

Fig. 2.-Radial velocities and galactic rotation.

The radial velocities, after correction for standard solar motion, were separated into three distance groups and means were formed for each $20^{\circ}$ of longitude. Stars closer than $300 \mathrm{pc}$, and more distant than $1800 \mathrm{pc}$, were excluded, the mean distances of the groups being 500,930 , and $1460 \mathrm{pc}$. The radial velocities were then analysed to obtain Oort's constant $A$ and a $K$ term by means of the equation,

$$
\rho=K+A r \sin 2 \lambda \text {, }
$$

where $\rho$ is the mean radial velocity and $\lambda$ is the longitude from the centre. Radial 
velocities of the interstellar $\mathrm{K}$-line of $\mathrm{Ca}^{+}$were analysed as well as the stellar velocities. In all, 406 stellar radial velocities were used and 284 velocities of the interstellar calcium.

Galactic rotation curves are shown in Figure 2 and the numerical values of $K$ and $A$ are given in Table 1.

TABLE 1

values of $K$ AND $A$

\begin{tabular}{|c|c|c|c|c|c|c|c|c|}
\hline \multirow{2}{*}{ Group } & \multicolumn{4}{|c|}{ Stellar Velocities } & \multicolumn{4}{|c|}{ Interstellar Velocities } \\
\hline & $\begin{array}{c}\bar{r} \\
(\mathrm{pc})\end{array}$ & $\begin{array}{c}K \\
(\mathrm{~km} / \mathrm{sec})\end{array}$ & $\begin{array}{c}A \\
(\mathrm{~km} / \mathrm{sec} . \mathrm{kpc})\end{array}$ & No. & $\begin{array}{c}\bar{r} \\
(\mathrm{pc})\end{array}$ & $\begin{array}{c}K \\
(\mathrm{~km} / \mathrm{sec})\end{array}$ & $\begin{array}{c}A \\
(\mathrm{~km} / \mathrm{sec} . \mathrm{kpc})\end{array}$ & No. \\
\hline I & 500 & $-2 \cdot 6 \pm 0 \cdot 7$ & $19 \cdot 8 \pm 1 \cdot 7$ & 195 & 525 & $-2 \cdot 4 \pm 0 \cdot 4$ & $8 \cdot 9 \pm 1 \cdot 0$ & 102 \\
\hline II & 930 & $-3 \cdot 3 \pm 0 \cdot 9$ & $16 \cdot 4 \pm 1 \cdot 3$ & 134 & 940 & $-0 \cdot 4 \pm 1 \cdot 1$ & $12 \cdot 1 \pm 1 \cdot 8$ & 110 \\
\hline III & 1460 & $-6 \cdot 0 \pm 2 \cdot 6$ & $19 \cdot 6 \pm 2 \cdot 3$ & 77 & 1470 & $-2 \cdot 6 \pm 0 \cdot 9$ & $10 \cdot 9 \pm 0 \cdot 8$ & 72 \\
\hline Means & & $-3 \cdot 0 \pm 0 \cdot 6$ & $17 \cdot 9 \pm 1 \cdot 2$ & & & $-2 \cdot 2 \pm 0 \cdot 4$ & $9 \cdot 2 \pm 0 \cdot 7$ & \\
\hline
\end{tabular}

The $K$ term is negative in all solutions with a mean value of about $3 \mathrm{~km} / \mathrm{sec}$, and the most distant group of stars suggests a more negative value. The behaviour is consistent with a small radial expansion of the Galaxy decreasing outward, as has been indicated by recent $21-\mathrm{cm}$ results. It is interesting that the radial velocities of the interstellar matter give a mean $K$ term in agreement with that from the stellar velocities.

The mean value of $A$ is $18 \mathrm{~km} / \mathrm{sec} . \mathrm{kpc}$ and the same value is derived from the interstellar velocities, if we assume that the effective distance of the interstellar material is one-half of the stellar distances. Our value of $A$ is in good agreement with that found by Feast and Thackeray (1958) from radial velocities of B stars but it is larger than the value of $15 \mathrm{~km} /$ sec.kpc recently deduced by Kraft and Schmidt (1963) from cepheid variables and Johnson and Svolopoulos (1961) from galactic clusters. A partial explanation of the difference may lie in the adoption, in this paper, of $20 \mathrm{~km} / \mathrm{sec}$ as the solar motion whereas Schmidt and $\mathrm{Kraft}$ found $16 \cdot 4 \mathrm{~km} / \mathrm{sec}$ from their material. The maximum of the galactic rotation curve, at $\lambda=45^{\circ}$, is near the longitude of the solar apex while the minimum, at $\lambda=135^{\circ}$, is nearly $90^{\circ}$ from the apex. Thus, if a solar motion larger than that appropriate to the B stars has been used, the result will be to increase the amplitude of the radial-velocity curves and hence to increase $\mathbf{A}$. This point will be investigated when the complete list of $\mathbf{B}$ stars is available for analysis.

The most powerful influence upon the numerical value of $A$ is, of course, the distance scale and it may be that distances based upon the Victoria calibration of $\mathbf{H}_{\gamma}$ are systematically too small. A direct comparison with the MK luminosity calibration (Petrie 1962) shows that $\mathrm{H}_{\gamma}$ distances are $15 \%$ less than those given by the luminosity classes and a similar difference is found in comparison with the photo- 
metric distance scale used for cepheids and galactic clusters. If the $\mathrm{H}_{\gamma}$ distances were to be increased by $15 \%$ the value of $A$ derived here would become $15 \mathrm{~km} / \mathrm{sec} . \mathrm{kpc}$. It is difficult to reach a decision upon corrections to the distance scales used for earlytype stars as these scales must still be subject to uncertainties. It is perhaps premature to attempt revisions of the scales but it is of interest to note that the constant $A$, derived here, would agree with those found from the cepheids and from galactic clusters were the same distance scales to be used.

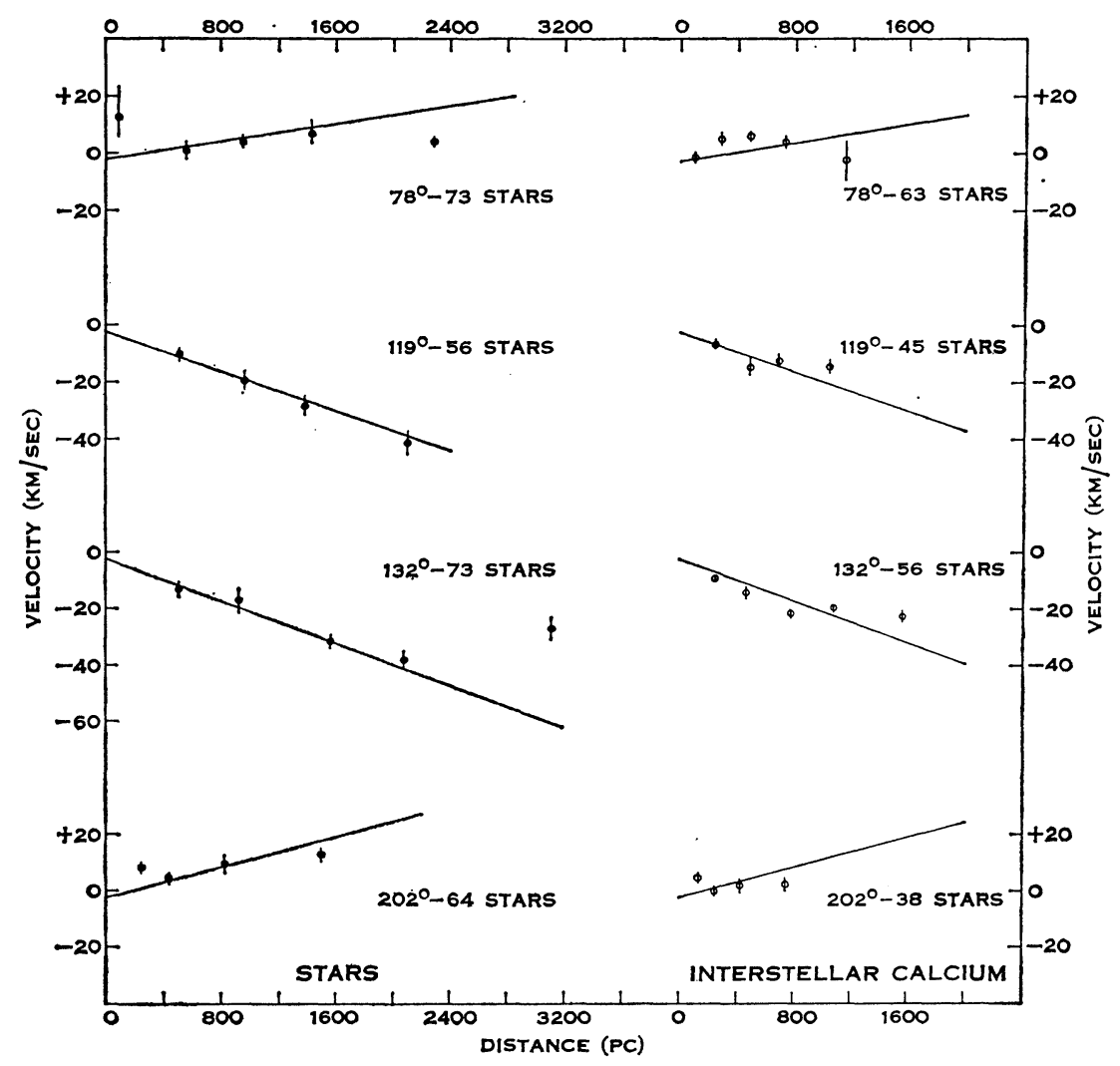

Fig. 3.-Velocity-distance relations at four selected longitudes.

Figure 3 shows plots of radial velocity versus distance at four selected longitudes. Such plots avoid the averaging effect, with longitude, of the conventional solution and allow one to utilize star groups at distances greater than those entering the solutions discussed above. The lines drawn in the diagram give the velocitydistance relations for a $K$ term of $-2.5 \mathrm{~km} / \mathrm{sec}$ and for $A=18 \mathrm{~km} / \mathrm{sec} . \mathrm{kpc}$.

The mean velocities plotted in Figure 3 conform in most cases to the required linear relation but there are some significant departures. The most spectacular of these is the deviation of $+33 \mathrm{~km} / \mathrm{sec} \pm 2 \cdot 0$ exhibited by a group of six stars in the direction of Perseus and at a distance of $3 \cdot 1 \mathrm{kpc}$, i.e. beyond the Perseus arm. One notes also that the velocities in the direction $\lambda=202^{\circ}$ show a smaller than 
expected increase with distance. The effects are presumably caused by systematic peculiar motions of groups of stars and might well be investigated by means of additional observations.

\author{
References \\ Feast, M. W., and Thackeray, A. D. (1958).-M.N. 118: 125-53. \\ Johnson, H. L., and Svolopoulos, S. N. (1961).-Ap.J. 134 : 868-73. \\ KERR, F. J. (1962).-M.N. 123 : 327-45. \\ Kraft, R. P., and SсHмidt, M. (1963).-Ap.J. $137: 249-67$. \\ Petrie, R. M. (1962).-M.N. 123 : 501-8. \\ Petrie, R. M., and Pearce, J. A. (1962).-P. Dom. Ap. O. 12 : No. 1.
}

Discussion

See Discussion on Paper No. 14.

\title{
14. FAINT SOUTHERN B STAR VELOCITIES
}

\section{A. D. Thackeray}

\section{Radcliffe Observatory}

At the Radcliffe Observatory, Dr. Feast and I have recently compiled a third list of radial velocities of $\mathrm{O}$ and $\mathrm{B}$ stars which was designed primarily to push our optical knowledge of the dynamics of the Galaxy to great distances from the Sun (Feast and Thackeray 1963). This program was preceded by the determination of distance moduli of $248 \mathrm{O}$ and B stars in cooperation with Dr. Stoy of the Royal Observatory, Cape, and Dr. Wesselink at the Radcliffe Observatory (Feast et al. 1961). As a result of this work we now have radial velocities for a very considerable number of southern stars at distances greater than 2 and even $3 \mathrm{kpc}$. It is quite possible that many of these distances are overestimated and it is highly desirable that the distances should be improved by the Walraven multicolour method or by some other technique. In the meantime it can certainly be claimed that our knowledge of radial velocities of these distant stars is considerably better in the southern than in the northern hemisphere.

There are three southern directions where radial velocities are of especial interest:

(1) The node near $l^{\mathrm{II}}=270^{\circ}$, from which a determination of $R_{0}$ can be made;

(2) the region of $l \mathrm{II}= \pm 30^{\circ}$, where according to the Leiden $\omega(R)$ curve the velocities increase almost linearly with distance out to 5 or $6 \mathrm{kpc}$;

(3) the direction to the centre, where it is specially important to compare velocities of stars and gas with the $21-\mathrm{cm}$ results.

Determination of $R_{0}$

In the predicted galactic rotation curve near the node at $l=270^{\circ}$ for stars at distance $2 \cdot 0,3 \cdot 2$, and $5 \cdot 0 \mathrm{kpc}$, there is of course a progressive shift of the node from $270^{\circ}$. It is also of interest that between 290 and $300^{\circ}$ the change in velocity predicted for stars from 2 to $5 \mathrm{kpc}$ is less than the intrinsic velocity dispersion of $\mathrm{B}$ stars. 